JOURNAL DE PHYSIQUE IV

Colloque C8, supplément au Journal de Physique III, Volume 6, décembre 1996

\title{
Diatomic Hydrogen-Metal Complexes in Dilute Palladium-Rare Earth Alloys
}

\author{
M. Ege, W. Ulfert and H. Kronmüller \\ Max-Planck-Institut für Metallforschung, Institut für Physik, Heisenbergstr. 1, 70569 Stuttgart, Germany
}

\begin{abstract}
The internal friction of hydrogen-doped dilute $\mathrm{Pd}_{100-x} \mathrm{RE}_{x}$ alloys $(\mathrm{RE}=\mathrm{Gd}$, Ce) $(x=1 \ldots 9)$ shows three maxima in the temperature range between $10 \mathrm{~K}$ and $300 \mathrm{~K}$. These maxima are interpreted as Zener peak of hydrogen in the $\beta$-phase $\left(H_{\mathrm{a}}=0.11 \mathrm{eV}\right)$, Snoek-Köster peak $\left(H_{\mathrm{a}}=0.18 \mathrm{eV}\right)$ and reorientation peak of diatomic $\mathrm{H}-\mathrm{RE}$ complexes. The relaxation strength of the latter peak is independent of the RE concentration and depends linearly on the hydrogen content. A value of $\left|\lambda_{1}-\lambda_{2}\right|=0.036$ for the elastic anisotropy of the H-RE complexes is obtained. In alloys with $\mathrm{RE}=\mathrm{Gd}$ this peak saturates when the hydrogen content is comparable with the $\mathrm{RE}$ concentration, whereas in the case of $\mathrm{RE}=\mathrm{Ce}$ saturation at much lower hydrogen contents is observed. The activation enthalpy $H_{\mathrm{a}}$ of the $\mathrm{H}-\mathrm{RE}$ reorientation varies with the $\mathrm{RE}$ as well as with the $\mathrm{H}$ concentration and lies between $0.18 \mathrm{eV}$ and $0.26 \mathrm{eV}$. The activation enthalpy and the width of the peak increase with increasing RE content. Increasing the $\mathrm{H}$ content leads to a decrease of $H_{\mathrm{a}}$. These features of the reorientation of diatomic H-RE complexes are discussed in terms of a distribution of site enthalpies caused by elastic long-range interaction between a H-RE complex and further RE atoms in its vicinity.
\end{abstract}

\section{Introduction}

The introduction of substitutional atoms into a cubic host lattice produces anisotropic interstitial sites. Hydrogen atoms on such sites are capable of showing relaxation processes. Jumps of a hydrogen atom around a substitutional atom lead to the reorientation of the axis of the hydrogen-substitutional atom pair (H-S pair). Such a reorientation of $\mathrm{H}-\mathrm{S}$ pairs has been observed in a large number of ferromagnetic alloys of b.c.c. as well as f.c.c. crystal structure $[1,2]$ using the measuring technique of the magnetic after-effect. The observation of the reorientation of $\mathrm{H}-\mathrm{S}$ pairs in b.c.c. host metals by means of internal friction (IF) is also well established $[3,4]$.

In contrast, observations of such kind in f.c.c. host metals were only recently reported for dilute Pd-Y alloys [5]. The IF of hydrogen doped samples of these alloys showed a Zener and a SnoekKöster peak, both of them well known in hydrogen-doped pure palladium. Additionally, these alloys exhibited a third peak which has no counterpart in pure palladium and which was attributed to the reorientation of $\mathrm{H}-Y$ pairs. In the present study, results for further palladium alloys, namely $\mathrm{Pd}-\mathrm{Gd}$ and Pd-Ce, will be presented.

\section{Experimental Procedure}

The relaxation of $\mathrm{H}$ in dilute $\mathrm{Pd}$-Ce alloys $\mathrm{Pd}_{100-x} \mathrm{Ce}_{x}(x=1 ; 5)$ and $\mathrm{Pd}-\mathrm{Gd}$ alloys $\mathrm{Pd}_{100-x} \mathrm{Gd}_{x}$ $(x=1 ; 2 ; 5 ; 9)$ has been studied by measurements of the IF. An inverted torsion pendulum (frequency $f=1 \ldots 10 \mathrm{~Hz}$ ) and a vibrating reed apparatus were used for the measurements. The alloys were prepared by arc-melting the elements (Pd: $99.99 \mathrm{wt} \%$, Gd,Ce: $99.9 \mathrm{wt. \%}$ ) in an argon atmosphere. The alloys were melted several times and subsequently annealed in high vacuum for $72 \mathrm{~h}$ at a temperature of $1273 \mathrm{~K}$. The homogeneity of the alloys was confirmed by scanning electron microscopy. Afterwards the bars were hammered down to wires of $3.5 \mathrm{~mm}$ or $2.0 \mathrm{~mm}$ in diameter respectively, followed by another high-vacuum annealing procedure at $1273 \mathrm{~K}$ for $72 \mathrm{~h}$. The samples used in the inverted torsion pendulum were of cylindrical shape with $2.0 \mathrm{~mm}$ in diameter and a length of about $30 \mathrm{~mm}$. The samples for the vibrating reed apparatus were obtained from wires with 
$3.5 \mathrm{~mm}$ in diameter. The vibrating part of these samples was prepared by spark erosion to the shape of a rectangular parallelepiped with a length of about $20 \mathrm{~mm}$, a width of $3.5 \mathrm{~mm}$ and a thickness varying between $0.4 \mathrm{~mm}$ and $1.8 \mathrm{~mm}$. The resulting vibration frequencies were between $450 \mathrm{~Hz}$ and $2100 \mathrm{~Hz}$.

The samples were doped with hydrogen by exposing them to $\mathrm{H}_{2}$ atmospheres of pressures ranging from $0.1 \mathrm{hPa}$ to $150 \mathrm{hPa}$. Doping temperature and time were $T=350 \mathrm{~K}$ and $t=24 \mathrm{~h}$ respectively. The hydrogen content was determined by measuring the increase of mass of the samples during doping. Prior to every doping procedure the samples were degassed in high vacuum at $1273 \mathrm{~K}$ for $12 \mathrm{~h}$ and subsequently chemically polished to remove a thin oxide layer from the surface.

\section{Experimental Results and Discussion}

\subsection{Pd-Gd alloys}

The internal friction of H-doped $\mathrm{Pd}_{100-x} \mathrm{Gd}_{x}$ is shown in figure 1. Fig.1a gives the results for the alloy $\mathrm{Pd}_{99} \mathrm{Gd}_{1}$, fig.1b those for $\mathrm{Pd}_{91} \mathrm{Gd}_{9}$. For the hydrogen content $c$ the notation $\mathrm{Pd}_{100-x} \mathrm{Gd}_{x} \mathrm{H}_{c}$ is used, indicating a sample that contains $c$ hydrogen atoms per hundred metal atoms.

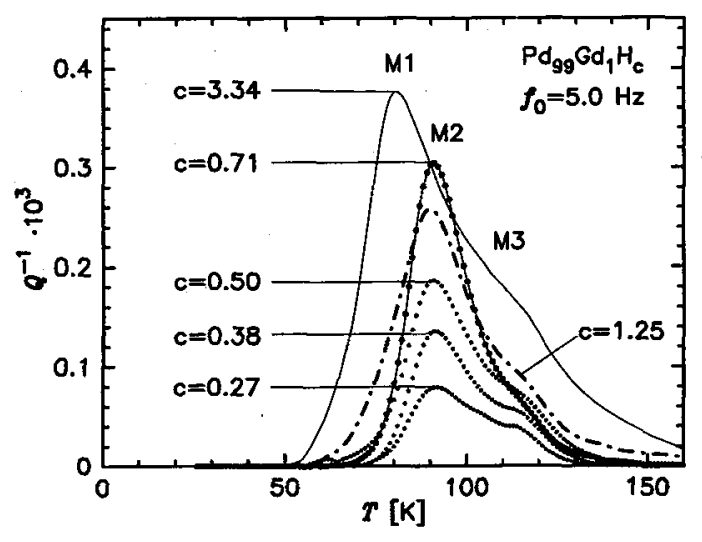

a)

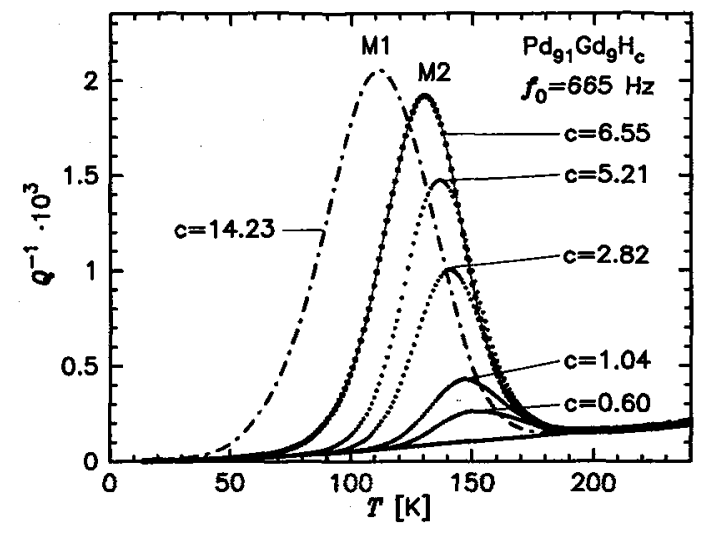

b)

Figure 1: IF spectra of hydrogen-doped dilute Pd-Gd alloys: a) $\mathrm{Pd}_{99} \mathrm{Gd}_{1} \mathrm{H}_{c}$ as measured in a torsion pendulum, background subtracted; b) $\mathrm{Pd}_{91} \mathrm{Gd}_{9} \mathrm{H}_{c}$ as measured in a vibrating reed apparatus.

Whereas the undoped alloys only show background damping without any maximum, $\mathrm{Pd}_{100-x} \mathrm{Gd}_{x} \mathrm{H}_{c}$ samples exhibit three different maxima that are denoted by $\mathrm{M} 1$, M2 and M3, respectively. As can be seen in fig.1, their occurrence depends on the $\mathrm{H}$ as well as on the Gd content. On the high temperature side of M2 a maximum M3 is observed. M3 is only weakly marked in undeformed samples but its relaxation strength raised by about an order of magnitude after a small plastic deformation in torsion. M3 was not observed in alloys containing more Gd than $x=2$ (fig.1b). For low $\mathrm{H}$ contents the height of the maximum M2 increases with increasing $c$ and saturates at a $H$ concentration $c^{s}$ that is comparable with the Gd concentration $x$ (table 2).

Further increasing of $c$ results in a decrease of $\mathrm{M} 2$ as can be seen for $c=1.25$ in fig.1a accompanied by the occurrence of another maximum M1 on the low temperature side of M2. This maximum M1 then increases monotonically with increasing $H$ content and dominates the IF spectrum for high values of $c$. The activation parameters of the relaxation time $\tau=\tau_{0} \cdot \exp \left(H_{\mathrm{a}} / k T\right)$ as determined from the frequency shifts in the torsion pendulum are given in table 1.

On the basis of these properties and activation parameters the maxima M1 and M3 can be interpreted as Zener relaxation of $H$ in the $\beta$-phase and as Snoek-Köster relaxation respectively. This interpretation gives an excellent agreement of the determined activation parameters in the Pd-Gd alloys with those of the Zener and Snoek-Köster relaxation in pure H-doped Pd [6].

The maximum M2 which has no counterpart in pure Pd is attributed to the reorientation of diatomic $\mathrm{H}-\mathrm{Gd}$ complexes. This interpretation is in keeping with the one for the Pd-Y system $[5,6]$ and makes it possible to understand the features of the maximum M2 as shown below. 
Table 1: Activation parameters of the three relaxation maxima in H-doped dilute Pd-Gd alloys $\mathrm{Pd}_{100-x} \mathrm{Gd}_{x} \mathrm{H}_{c}(x=1 \ldots 9)$. The value of $H_{\mathrm{a}}$ of M2 depends on $x$ and $c$.

\begin{tabular}{|c|c|c|c|}
\hline Maximum & $\tau_{0}[\mathrm{~s}]$ & $H_{\mathrm{a}}[\mathrm{eV}]$ & Process \\
\hline \hline M1 & $2 \cdot 10^{-9 \pm 0.5}$ & $0.11( \pm 0.01)$ & Zener relaxation \\
\hline M2 & $3 \cdot 10^{-12 \pm 0.5}$ & $0.18 \ldots 0.26( \pm 0.01)$ & H-Gd reorientation \\
\hline M3 & $4 \cdot 10^{-10 \pm 0.5}$ & $0.18( \pm 0.01)$ & Snoek-Köster relaxation \\
\hline
\end{tabular}

Table 2: Saturation concentration $c^{5}$ of the $\mathrm{H}-\mathrm{Gd}$ reorientation maximum (M2) for dilute Pd-Gd alloys.

\begin{tabular}{|c||c|c|c|c|}
\hline Alloy & $\mathrm{Pd}_{99} \mathrm{Gd}_{1}$ & $\mathrm{Pd}_{98} \mathrm{Gd}_{2}$ & $\mathrm{Pd}_{95} \mathrm{Gd}_{5}$ & $\mathrm{Pd}_{91} \mathrm{Gd}_{9}$ \\
\hline \hline$c^{\mathrm{s}}$ & $\mathbf{0 . 7 1}$ & 1.64 & 4.51 & $\mathbf{6 . 5 5}$ \\
\hline
\end{tabular}

Whereas the pre-exponential factor $\tau_{0}$ of M2 does not show any significant dependence on $x$ or $c$, the activation enthalpy $H_{\mathrm{a}}$ of the $\mathrm{H}-\mathrm{Gd}$ relaxation increases with the Gd content. Furthermore, a decrease of $H_{\mathrm{a}}$ is observed with increasing $\mathrm{H}$ content $c$ which is particularly marked in alloys with higher Gd content and results in a shift of the maximum to lower temperatures for higher values of $c$ (fig.1b). For all the alloys studied, maximum M2 is broader than a single Debye maximum and its width increases with the Gd content (fig.1).

These properties of the reorientation of $\mathrm{H}-\mathrm{Gd}$ pairs are explained by a distribution of site enthalpies for the $\mathrm{H}$ atoms forming $\mathrm{H}-\mathrm{Gd}$ pairs. The origin of this distribution is the elastic long-range interaction between the $\mathrm{H}$ atom of a $\mathrm{H}-\mathrm{Gd}$ complex and further $\mathrm{Gd}$ atoms in its environment. Details of the calculation of this distribution are given in a previous paper [5]. The obtained distributions are very sharp for highly dilute alloys $(x=1)$ (isolated $\mathrm{H}$-Gd complexes), whereas in the case of higher substitutional contents broader distributions with contributions of lower site enthalpies result. Using the assumption of nearly constant saddle-point enthalpy for the reorientation jumps of the $\mathrm{H}$ atoms the experimentally observed features of the $\mathrm{H}-\mathrm{Gd}$ reorientation maximum can be explained by the aid of the distributions of site enthalpies being successively filled up with $H$ atoms [5].

To account for the broadening of the maximum attributed to the reorientation of H-Gd complexes a superposition of Debye maxima is assumed, applying a spectrum of relaxation times with a constant pre-exponential factor $\tau_{0}=3 \cdot 10^{-12} \mathrm{~s}$. Details of this procedure have been given elsewhere [5]. As a result of this analysis the total relaxation strength $\Delta$ of the maximum is obtained in dependence of the hydrogen content $c$. Figure 2 gives the results for $\Delta$ taking into consideration only measurements with hydrogen contents lower than the corresponding saturation concentration $c^{s}$.

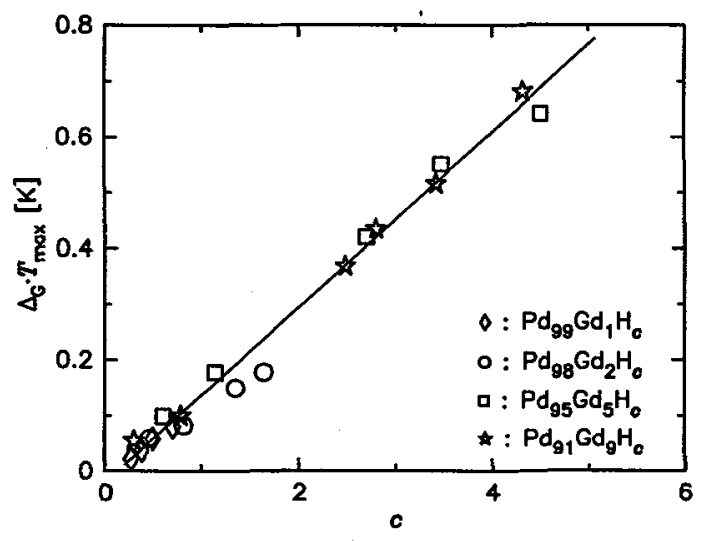

a)

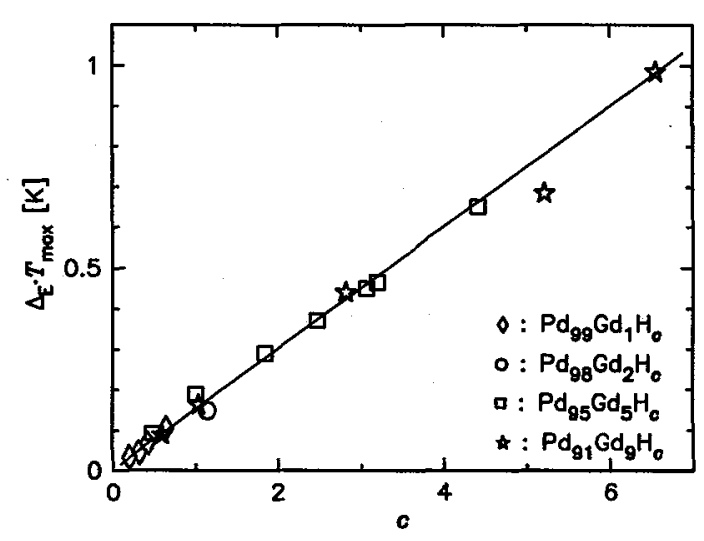

b)

Figure 2: Dependence of the relaxation strength $\Delta$ of the H-Gd reorientation on hydrogen content $c$, as determined by analysis of IF measurements in a) torsion and b) bending mode. 
For all the alloys studied, the relaxation strength $\Delta$ depends linearly on the hydrogen concentration $c$, the slope of the straight line being independent of the Gd content (fig.2). This fact indicates that the formation of $\mathrm{H}$-Gd complexes is not statistical but energetically favoured. In fact, applying a model for the formation of hydrogen-substitutional atom pairs in transition metals [7] indicates that substitutional $\mathrm{Gd}$ atoms in a Pd matrix should act as trapping centers for interstitial $\mathrm{H}$ atoms.

Assuming that the binding energy $E_{\mathrm{b}}$ of these diatomic H-Gd complexes (a minimum value of $E_{\mathrm{b}}=0.06 \mathrm{eV}$ can be estimated from fig.2, [8]) is high enough for all $\mathrm{H}$ atoms to form complexes (as long as $c<c^{s}$ ) and that the symmetry of these complexes is tetragonal, the following relation between the relaxation strength $\Delta$ and the hydrogen content $c$ holds:

$$
\Delta=\beta \frac{c}{100} \frac{v_{0}}{k T_{\max }} M_{\text {poly }}\left(\lambda_{1}-\lambda_{2}\right)^{2}
$$

Here, $k$ is the Boltzmann constant, $T_{\max }$ the temperature of the maximum, $v_{0}$ the atomic volume of the Pd lattice and $\beta$ a numerical factor. The shear modulus $G_{\text {poly }}$ (and $\beta=4 / 15$ ) and the Young modulus $E_{\text {poly }}$ (and $\beta=4 / 45$ ) have to be used for $M_{\text {poly }}$ when the peak is observed in torsion and in flexure, respectively. For the elastic anisotropy $\left|\lambda_{1}-\lambda_{2}\right|$ of the H-Gd complexes values of 0.035 and 0.037 are obtained from fig.2a and $2 b$ respectively. The mean value of 0.036 is the same as for H-Y complexes [5].

\subsection{Pd-Ce alloys}

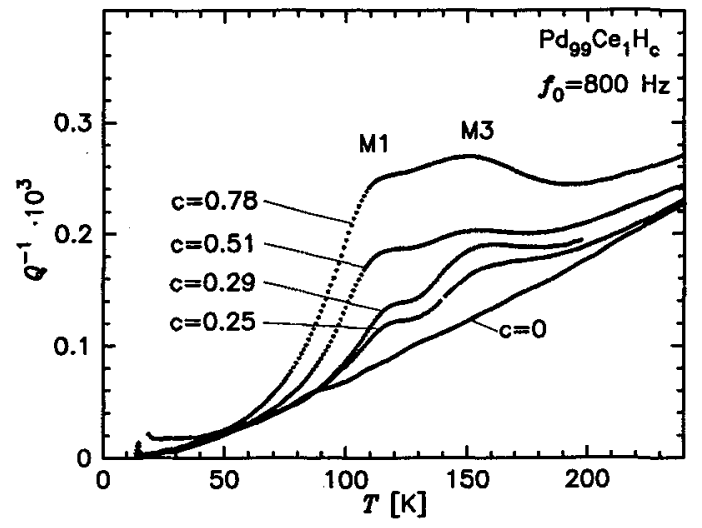

a)

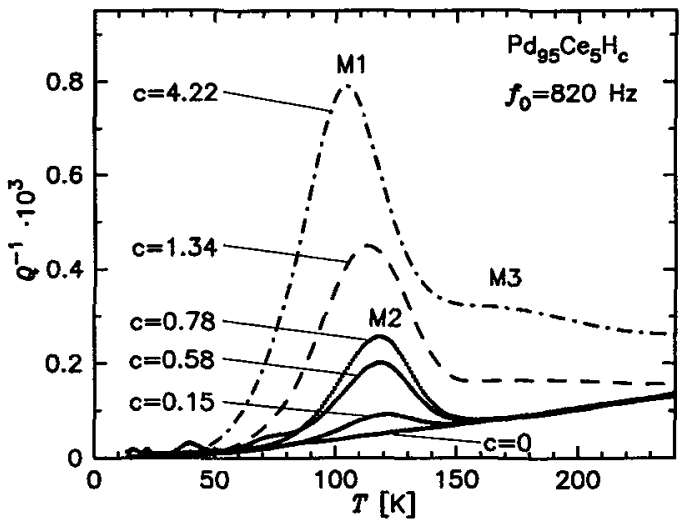

b)

Figure 3: IF spectra of hydrogen-doped dilute $\mathrm{Pd}$-Ce alloys: a) $\mathrm{Pd}_{99} \mathrm{Ce}_{1} \mathrm{H}_{c}$ and b) $\mathrm{Pd}_{95} \mathrm{Ce}_{5} \mathrm{H}_{c}$ as measured in a vibrating reed apparatus.

The measurements of the IF of $\mathrm{Pd}_{100-x} \mathrm{Ce}_{x} \mathrm{H}_{c}(x=1 ; 5)$ are shown in fig. 3 . In contrast to the results on Pd-Gd alloys, in $\mathrm{H}$-doped $\mathrm{Pd}$-Ce alloys the maximum M2 is not so clearly marked. In $\mathrm{Pd}_{99} \mathrm{Ce}_{1} \mathrm{H}_{c}$ only the Zener and the Snoek-Köster maximum (M1 and M3 respectively in fig.3a) are observed. Measurements on $\mathrm{Pd}_{95} \mathrm{Ce}_{5} \mathrm{H}_{c}$ with higher Ce content show a maximum (M2 in fig. $3 \mathrm{~b}$ ) that occurs only for low $\mathrm{H}$ contents up to $c=0.78$. This maximum has comparable activation parameters as the maximum M2 in Pd-Gd and is therefore attributed to the reorientation of H-Ce complexes. The fact that this maximum occurs only for low $\mathrm{H}$ contents in Pd-Ce may be explained by merely a small binding energy of Pd-Ce complexes.

\section{References}

[1] Kronmüller H., Higelin G., Vargas P. and Lässer R., Z. Phys. Chem. NF 143 (1985) 161-181

2 Hohler B. and Kronmüller H., Z. Phys. Chem. NF 114 (1979) 93-108

3 Cannelli G., Cantelli R. and Koiwa M., Phil. Mag. A 46 (1982) 483-494

4 Cannelli G., Cantelli R., Cordero F. and Trequattrini F., Mater. Sci. Forum 119-121 (1993) 29-38

5 Ege M., Hauptmann G., Ulfert W. and Kronmüller H., J. Alloys Compd. 211/212 (1994) 222-225

6] Hauptmann G., Ulfert W. and Kronmüller H., Mater. Sci. Forum 119-121 (1993) 109-114

7 . Vargas P., Kronmüller H. and Böhm M.C., Z. Phys. Chem. NF 143 (1985) 229-245

[8] Ege M. and Kronmüller H., to be published 